\title{
RELEVANCE OF DIAGNOSTIC INVESTIGATIONS IN CHRONIC INFLAMMATORY DEMYELINATING POLIRADICULONEUROPATHY: DATA FROM THE ITALIAN CIDP DATABASE
}

Giuseppe Liberatore, ${ }^{1}$ Fiore Manganelli, ${ }^{2}$ Dario Cocito, ${ }^{3}$ Raffaella Fazio, ${ }^{4}$ Chiara Briani, ${ }^{5}$ Massimiliano Filosto, ${ }^{6}$ Luana Benedetti, ${ }^{7,8}$ Giovanni Antonini, ${ }^{9}$ Giuseppe Cosentino, ${ }^{10}$ Stefano Jann, ${ }^{11}$ Anna Mazzeo, ${ }^{12}$ Andrea Cortese, ${ }^{13}$ Girolama Alessandra Marfia, ${ }^{14}$ Angelo Maurizio Clerici, ${ }^{15}$ Gabriele Siciliano, ${ }^{16}$ Marinella Carpo,${ }^{17}$ Mario Sabatelli, ${ }^{18,19}$ Giuseppe Lauria, ${ }^{20,21}$ Tiziana Rosso, ${ }^{22}$ Eduardo Nobile Orazio ${ }^{1,23}$ on the behalf of the Italian CIDP Database Study Group.

1. Neuromuscular and Neuroimmunology Service, Humanitas Clinical and Research Institute, Rozzano, Milan, Italy

2. Department of Neuroscience, Reproductive Sciences and Odontostomatology, University of Naples 'Federico II', Naples, Italy

3. Presidio Sanitario Major, Istituti Clinici Scientifici Maugeri, Turin, Italy

4. Division of Neuroscience, Department of Neurology, Institute of Experimental Neurology (INSPE), San Raffaele Scientific Institute, Milan, Italy

5. Neurology Unit, Department of Neuroscience, University of Padua, Padua, Italy

6. Center for Neuromuscular Diseases and Neuropathies, Unit of Neurology, ASST 'Spedali Civili', University of Brescia, Brescia, Italy

7. Department of Neuroscience, Rehabilitation, Ophthalmology, Genetics, Maternal and Child Health, University of Genoa and IRCCS AOU San Martino-IST, Genoa, Italy

8. Neurology Unit, Sant'Andrea Hospital, La Spezia, Italy

9. Unit of Neuromuscular Diseases, Department of Neurology Mental Health and Sensory Organs (NESMOS), Faculty of Medicine and Psychology, 'Sapienza' University of Rome, Sant'Andrea Hospital, Rome, Italy

10. Department of Experimental BioMedicine and Clinical Neurosciences (BioNeC), University of Palermo, Palermo, Italy

11. Department of Neuroscience, Niguarda Ca' Granda Hospital, Milan, Italy

12. Department of Clinical and Experimental Medicine, Unit of Neurology, University of Messina, Messina, Italy

This is the author manuscript accepted for publication and has undergone full peer review but has not been through the copyediting, typesetting, pagination and proofreading process, which may lead to differences between this version and the Version of Record. Please cite this article as doi: $10.1111 /$ jns.12378

This article is protected by copyright. All rights reserved. 
13. IRCCS Foundation C. Mondino National Neurological Institute, Pavia, Italy

14. Dysimmune Neuropathies Unit, Department of Systems Medicine, Tor Vergata University of Rome, Rome, Italy

15. Neurology Unit, Circolo \& Macchi Foundation Hospital, Insubria University, DBSV, Varese, Italy

16. Neurology Unit, Department of Clinical and Experimental Medicine, University of Pisa, Pisa, Italy

17. Neurology Unit, ASST Bergamo Ovest-Ospedale Treviglio, Treviglio, Italy

18. NEuroMuscular Omnicentre (NEMO), Serena Onlus Foundation - Pol. A. Gemelli, Rome, Italy

19. Catholic University of Sacred Heart, Rome, Italy.

20. Unit of Neuroalgology, IRCCS Foundation 'Carlo Besta' Neurological Institute, Milan, Italy

21. Department of Biomedical and Clinical Sciences 'Luigi Sacco', University of Milan, Milan, Italy

22. ULSS2 Marca Trevigiana, UOC Neurologia-Castelfranco Veneto, Treviso, Italy

23. Department of Medical Biotechnology and Translational Medicine, Milan University, Milan, Italy

Address Correspondence to: Giuseppe Liberatore, MD, Neuromuscular and Neuroimmunology Service, Humanitas Clinical and Research Institute, Via Manzoni 56, Rozzano, Milan 20089, Italy. Tel: +390282247243; Fax: +390282242298; E-mail: giuseppe.liberatore@humanitas.it

Submission Type: Original article

Running title: Clinical features and diagnostic investigations in CIDP

Number of Tables: $\mathbf{3}$

Number of Figures: 2

Word count of Abstract: 254

Word Count of Paper: 2898 


\section{ABSTRACT}

Background and aims: to report the clinical features and the relevance of diagnostic investigations in patients with chronic inflammatory demyelinating polyradiculoneuropathy (CIDP).

Methods: We retrospectively reviewed data from patients with a clinical diagnosis of CIDP included in a national database.

Results: Among the 500 included patients with a clinical diagnosis of CIDP, 437 patients (87\%) fulfilled the EFNS/PNS criteria for CIDP (definite in 407, probable in 26, possible in four). In 352 patients (86\%) motor nerve conduction abnormalities consistent with demyelination were sufficient for the diagnosis of definite CIDP. In 55 patients this diagnosis required the addition of one or two (from probable or from possible CIDP, respectively) supportive tests, while in 20 cases they improved the diagnosis from possible to probable CIDP, seven patients did not change diagnosis. Considering these 85 patients, CSF studies were performed in 79 cases (93\%) upgrading the certainty of diagnosis in $59 \%$ of examined patients. Sensory nerve conduction studies were performed in $85 \%$ of patients with an improvement of diagnosis in $32 \%$ of cases. Nerve biopsy and US/MRI exams resulted positive in about $40 \%$ of examined patients, but they were performed in few patients (7 patients and 16 patients, respectively). A response to therapy was present in $84 \%$ of treated patients $(n=77)$, contributing to support the diagnosis in 40 patients in whom the other supportive criteria were not sufficient.

Conclusions: In most patients with CIDP the diagnosis is possible solely with motor nerve conduction studies while other investigations may help improving the diagnosis in a minority of patients. 
Keywords: Chronic inflammatory demyelinating neuropathy; CIDP; Peripheral neuropathy; EMG;

Diagnostic criteria.

This article is protected by copyright. All rights reserved. 


\section{INTRODUCTION}

Chronic inflammatory demyelinating polyneuropathy (CIDP) is the most common chronic acquired demyelinating polyneuropathy with a prevalence ranging from 1 to 9 cases per $100000^{1,2}$. CIDP is considered an immune-mediated disease and often improves after immunotherapies ${ }^{3-5}$. Typically, the clinical picture includes mostly symmetric proximal and distal muscle weakness, sensory loss, and decreased or absent deep tendon reflexes. The disease course is steadily or stepwise progressive over at least 2 months, or relapsing ${ }^{3-5}$. Several variants have been described based on distribution of symptoms and signs ${ }^{3-5}$.

Diagnosis of CIDP can be challenging and, in recent years, several different sets of diagnostic criteria have been created with variable combinations of electrophysiological and/or clinical features $^{6-9}$. Currently, the most widely accepted criteria are those recommended by the European Federation of Neurological Societies and Peripheral Nerve Society (EFNS/PNS) ${ }^{9}$, that were shown to provide the best combination of sensitivity and specificity (about $75 \%$ and $90 \%$, respectively) for the diagnosis of CIDP compared with the other criteria ${ }^{10-11}$. A number of additional diagnostic investigations have been included in these criteria to support the diagnosis in patients not fulfilling the electrodiagnostic criteria. The relative diagnostic relevance of these investigations remains unclear and they are often unnecessarily performed in these patients.

We reviewed the data from a large cohort of patients included in the Italian CIDP database with the aim to analyze the clinical features, diagnostic investigations and response to therapy in patients with CIDP and to analyze the contribute of additional investigations in improving the diagnostic certainty. 


\section{PATIENTS AND METHODS}

\section{Database and study population}

This was a retrospective multicenter cohort study in large sample of prevalent CIDP patients in Italy, with additional follow-up data scheduled up to 2 years from enrolment. We implemented a web-based database (CINECA, Bologna, Italy) to collect data from patients with CIDP followed by 21 centers throughout Italy with expertise on CIDP. All CIDP patients visiting outpatient clinics of the participating neuromuscular expert centers were eligible for inclusion. Clinical and diagnostic data were obtained by experienced neurologist with a neuromuscular subspecialty. Verification of the diagnostic data for all of the enrolled patients were centralized in the principle study center (Humanitas), and based on expert panel consensus. Data monitoring included diagnosis revision, suspect double entries, missing data and plausibility checks. We excluded patients with an alternative diagnosis for the neuropathy, increased titers of anti-myelin-associated glycoprotein (MAG) IgM antibodies (over 7000 Unit by Buhlman method in our laboratory) or without available nerve conduction studies (NCS).

The study was approved by the Ethical Committee of each participating Center. All the patients gave written informed consent.

\section{Clinical assessment and ancillary tests}

The patients were subjected to detailed clinical history including duration of weakness, sensory symptoms, ataxia, pain, autonomic dysfunction. The course of the disease was defined by the treating neurologist as progressive or relapsing. A relapsing course was defined as a clinical worsening of the patient that was not related to a suspension or reduction of the dose of therapy. An 
acute (GBS-like) onset was also reported and defined as a neuropathy that was initially diagnosed as GBS but that continued to progress or relapsed after more than two months from disease onset. Response to previously performed therapy was reported by the treating neurologist and defined as improvement, stability or worsening. Response to therapies was defined as an improvement of $\geq 2$ points at MRC scale or an improvement of $\geq 1$ point at INCAT scale.

The clinical evaluation at enrollment included assessment of muscle strength with the Medical Research Council (MRC) scale ${ }^{12}$, range 1-60, sensory function with the INCAT sensory sum score (ISS) ${ }^{13}$, range $1-20$, and neurological disability with INCAT scale ${ }^{14}$, range $0-10$. Cranial nerve involvement included assessment of III, IV, V, VI, VII, VIII, IX, X nerves, either considering a partial or complete paralysis, either unilateral or bilateral involvement.

Results of cerebrospinal fluid (CSF) examination performed during the course of the disease, including total protein level and cell counts, were reported. As to protein counts, we considered as upper reference limit $50 \mathrm{mg} / \mathrm{dl}$ for patients aged $\leq 50$ years and $60 \mathrm{mg} / \mathrm{dl}$ for those aged $>50$ years ${ }^{15}$. Moreover, we collected the results of nerve ultrasound (US), considering as abnormal a sonographic enlargement of proximal median nerve segments in the arms and brachial plexus; MRI examination and sural nerve biopsy results were considered abnormal based on the indication of EFNS/PNS criteria9

The results of diagnostic NCS performed during the course of the disease were included. Motor nerve conduction studies were planned to be performed bilaterally in the median, ulnar, common peroneal and tibial nerves and included distal (measured at wrist for upper arms and at ankle for lower arms) and proximal (measured at elbow for upper arms and at fibular head or popliteal fossa for lower arms) compound muscle action potential (CMAP) amplitude (onset to peak) and duration, motor conduction velocities (MCV), distal and proximal motor latencies and in 
most patients F-wave latency. Sensory conduction studies were planned to be performed bilaterally in the median, ulnar and sural nerves and included sensory action potential (SAP) amplitude, distal latency (DL) and conduction velocity (SCV). Some patients also underwent somatosensory evoked potentials (SSEP). We used the electrodiagnostic criteria for demyelination proposed by the EFNS/PNS ${ }^{9}$. NCS data were centrally reviewed based on rater experience, taking in consideration a tabulated summary of results provided by each center.

\section{Statistical analysis}

Descriptive statistics were reported for the sample of patients with CIDP overall and separately for the subgroups with a relapsing or progressive course or an acute onset of CIDP. Categorical variables were described using frequencies and percentages, while continuous variables using mean, medians and ranges. Demographic and clinical features, including response to therapy, were compared between different subgroups of patients with the chi-square or the Fisher's exact test for categorical variables, and with the t-test or the Wilcoxon-Mann-Whitney test for continuous variables. Considering that each variable was analyzed at maximum 2 times, we considered as statistical significant a value of $\mathrm{p}=0.025(0.05 * 2)$, in order to reduce type I error. Analyses were performed with IBM SPSS Statistics for Windows, Version 19.0 (Armonk, NY: IBM Corp, USA).

\section{RESULTS}

From January 2015 to January 2019 we enrolled 545 patients in our database (Figure 1). Twentyfour patients were excluded from the analysis for the presence of a different cause for neuropathy and 21 patients for unavailable neurophysiological data. A total of 500 patients were included in the study. At study entry, 437 patients fulfilled the EFNS/PNS criteria including two patients with 
typical chronic immune sensory polyradiculopathy (CISP) ${ }^{16}$ and normal motor conduction studies. Sixty-three (13\%) patients had a medical history and clinical signs compatible with CIDP without fulfilling the EFNS/PNS electrodiagnostic criteria, but fulfilling at least one supportive criterion (Figure 1).

\section{Demographic and clinical features of patients fulfilling EFNS/PNS criteria for CIDP}

Of the 437 patients fulfilling the EFNS/PNS criteria, 282 were men (65\%) and 155 women (35\%) (ratio: 1.83:1) with a mean age at inclusion of 58.1 years (median 60; range 11-92), a mean age at onset of 49.9 years (median 51; range 6-86 years), a mean disease duration of 8.1 years (median 5; range 0.2-52 years) and a mean INCAT score at enrollment of 2.6 (median 2; range 0-10) (Table 1).

Even if the majority of patients had sensorimotor symptoms at onset (51.4\%), a consistent proportion of them had purely sensory (31.4\%) or motor symptoms (15.4\%) at onset while occasional patients presented with pain, diplopia or ataxia (Table1). Almost all patients with typical CIDP (98.4\%) had sensorimotor symptoms at the time of enrollment while in three patients motor symptoms were associated with pain and in four patients sensory symptoms with only motor cranial nerve involvement.

The diagnosis was of typical CIDP in 353 (80.8\%) patients and of atypical CIDP in 84 (19.2\%) according to our criteria ${ }^{17}$. The diagnosis of CIDP according to EFNS/PNS criteria was definite for 407 patients (93.1\%) and probable for 26 patients (6\%); only four patients (0.9\%) had a diagnosis of possible CIDP. Within the group of patients with definite CIDP, 352 patients (86.4\%) fulfilled definite electrodiagnostic criteria, while in 55 patients diagnosis of definite CIDP was obtained with the combination of electrodiagnostic and supportive criteria: 20 patients (4.9\%) with probable CIDP plus at least one supportive criteria, 35 patients (8.7\%) with possible CIDP plus at 
least 2 supportive criteria. Within the group of patients with probable CIDP, three patients fulfilled probable electrodiagnostic criteria, while 23 patients had a possible CIDP plus one supportive criteria. Consequently, we could improve the certainty of diagnosis by means of supportive criteria (from possible to definite, from probable to definite, from possible to probable) for 78 patients, while in 7 cases supportive criteria were not sufficient to improve diagnosis.

As to clinical course, a similar proportion of patients had a relapsing (53\%) or progressive (47\%) course, while in five patients the course was not specified (Table 1 and Table 2). Patients with a relapsing course were younger at onset and at enrollment than those with a progressive course. They also had a lower INCAT score, a trend towards a more frequent acute CIDP onset and towards a less frequent response to IVIg (Table 2).

In 41 patients (9\%) there was an acute onset of CIDP that was followed by a relapsing (68\%) or progressive course (32\%). Patients with an acute onset of CIDP were younger, had a trend towards a worse INCAT score at entry, a more frequent involvement of strength and cranial nerve and a more frequent response to therapy than patients without an acute onset (Table 3).

\section{Diagnostic investigations and response to therapy in patients fulfilling EFNS/PNS criteria for CIDP}

For 346 patients (86\%) the diagnosis of definite CIDP was possible through the presence of motor nerve conduction abnormalities consistent with demyelination according to the EFNS/PNS criteria (mean number of motor nerves examined 5.6, median 6, range 2-8). Abnormality consistent with demyelination in at least two motor nerves were found on conduction velocity in 177 patients (51\%), conduction block in 157 (45\%), increased temporal dispersion in 122 (35\%), increased distal latency in 69 (20\%) and increased minimal F-wave in 32 (9\%).

This article is protected by copyright. All rights reserved. 
Among the 85 patients with NCS-possible or NCS-probable CIDP who improved (78 patients) or not (seven patients) the certainty of their diagnosis with supportive criteria, 47 (55\%) had increased CSF proteins, 23 (27\%) demyelinating features on sensory NCS or evoked potential, three (3.5\%) demyelinating or inflammatory findings on nerve biopsy and seven (8\%) enlargement or enhancement of nerve, plexus or roots by ultrasound (US) or MRI (Figure 2). Response to immunotherapies was present in 65 patients (76.5\%); notably, in 40 patients (47\%) response to immune therapy improved diagnostic definition when the other supportive criteria were not sufficient. The evaluation of supportive criteria was not uniformly performed in these patients, so the ratios between positive patients/examined patients were as follows: increased CSF protein in 47/79 cases (59.4\%), sensory nerve demyelination in 23/72 cases (32\%), abnormal nerve biopsy in 3/7 cases (43\%), abnormal US/MRI in 7/16 cases (43.7\%), response to therapy in 65/77 cases (84.4\%) (Figure 2).

Among the total 437 CIDP patients, 336 (77\%) underwent CSF examinations showing increased CSF proteins in 230 (68\%, mean $106.1 \mathrm{mg} / \mathrm{dl}$; median $75 \mathrm{mg} / \mathrm{dl}$; $\geq 80 \mathrm{mg} / \mathrm{dl}$ in 47\% of patients); as to white cell count, only in 10 patients we found $>10$ cells/ul (higher value 45 cells/ul in one case). Sensory conduction or somato-sensory evoked potential (SSEP) studies were performed in 394 (90.2\%) patients, and demyelinating findings in 150 (38\%) patients (mean number of sensory nerves examined 3.7, median 4). We combined data on SSEP and nerve conduction studies since we had data on SSEP only for 27 patients so was not useful to analyze separately. Nerve biopsy was performed in 35 (8\%) patients showing signs of demyelination or inflammatory infiltrates in 21 (60\%); 65 (14.9\%) patients performed US or MRI and in 50 (77\%) cases there were nerve or roots enlargement or contrast enhancement. 
Improvement after any of the immune therapy used was observed in 338/393 (86\%) treated patients while 51 (13\%) treated patients remained stable and 5 (1\%) worsened despite therapy (Table 1). Improvement after therapy with high dose intravenous immunoglobulins (IVIg) was reported in 244/334 (73\%) treated patients and after oral or intravenous corticosteroids in 123/235 (52\%) treated patients. Only 43 patients were treated with plasma exchange with an improvement observed in 25 (52\%). Subcutaneous immunoglobulins (SCIg) were used in 65 patients previously treated with IVIg with improvement (43\%) or stability (48\%) in the majority of them. Immunosuppressive agents (including Azathioprine, Cyclophosphamide, Mycophenolate Mofetil, Methotrexate, Cyclosporine, Rituximab) were used in 97 patients with a reported improvement in 31 (32\%), including 12 patients in whom improvement was only related to this therapy (5 Rituximab, 2 Cyclophosphamide, 2 Mycophenolate and 3 Azathioprine).

\section{DISCUSSION}

Currently, the most used CIDP criteria in clinical trial and in clinical practice are EFNS/PNS criteria $^{9}$, since different comparison studies confirmed that they offer the best combination in terms of sensitivity/specificity. In these criteria a number of supportive investigations were included to improve the diagnostic certainty in patients not fulfilling the electrodiagnostic criteria. However, the relative diagnostic relevance of these investigations remains unclear. In addition, they do not allow a diagnosis of CIDP in patients not having a demyelinating feature in at least one motor nerve, so that they may only improve the diagnostic certainty in patients already fulfilling a possible or probable diagnosis.

We reviewed the data from 500 patients with a diagnosis of CIDP and its variants included in the Italian CIDP database. Within this group, 437 (87.4\%) patients had a diagnosis of CIDP 
according to EFNS/PNS criteria while 63 (12.6\%) patients, despite the presence of clinical signs and symptoms compatible with this diagnosis, did not fulfill ENFS/PNS criteria, confirming the reported sensitivity of these criteria ${ }^{10,11}$.

Our study provide some information on the frequency and clinical features of patients with CIDP in relation to the type of disease onset and clinical course. A similar proportion of patients had a progressive or relapsing course. Patients with a progressive course had a later age at onset and a more severe impairment compared to patients with a relapsing course despite a similar disease duration, confirming previously reported observations ${ }^{18}$. They also had a slightly more frequent response to IVIg than patients with a relapsing course. Patients with an acute onset of CIDP had a younger age at onset and a higher severity than patients without an acute onset. They also had a lower frequency of sensory symptoms at onset and a higher frequency of motor and cranial nerve involvement during the disease compared to patients without an acute onset. Similar differences were recently reported between patients with GBS and patients with acute-onset CIDP ${ }^{19}$ who were less impaired, had less frequent cranial nerve dysfunction and more frequent sensory disturbances, possibly suggesting that patients with an acute-onset CIDP may have some overlapping features between typical CIDP and GBS ${ }^{20}$.

In the vast majority of patients with CIDP, motor nerve conduction studies were sufficient to confirm the diagnosis. In 352 patients (80.5\%) with a definite diagnosis of CIDP this diagnosis was possible with only motor nerve conduction studies, while in 75 patients (17.1\%) supportive criteria helped to improve the diagnostic certainty. In order to exclude differences in the performed electrodiagnostic tests, we analyzed mean number of nerve tested among the three subgroup of definite, probable and possible CIDP and we found following value: 5.6 vs 5.3 vs 4.6 , respectively 
( $\mathrm{p}=0.001$ ). The difference was statistically significant but the mean number of nerve tested in the possible patients (4.6) can be considered clinically relevant to formulate a correct diagnosis.

CSF analysis (55\%) and sensory nerve conduction studies (27\%) were particularly helpful in improving the diagnosis while nerve biopsy and nerve US or MRI rarely improved the diagnosis. Those investigations were, however, performed in a much larger proportion of patients, particularly in the case of CSF studies performed in 336 patients (77\%), while nerve biopsy and nerve or roots US/MRI were performed in 35 patients (8\%) and 65 patients (15\%), respectively. In our opinion, it is not possible to infer data on sensitivity about biopsy, ultrasound and MRI because there could be a strong selection bias, since tests were performed in a very limited percentages of cases.

Overall, our findings support the opinion proposed by the EFNS/PNS that these studies should be probably restricted to patients in whom a definite diagnosis of CIDP is not reached with motor electrodiagnostic studies unless a possible alternative diagnosis is considered.

Limitations of this study include its retrospective nature with the possibility to have less accurate data on patients with a longer disease duration and the lack of a control sample of patients not affected with CIDP. Moreover, US/MRI and nerve biopsy are not routinely performed exams, so we have data from few patients. Data on response to therapy should be also considered with caution as they were mainly based on the clinicians' reports and patients' view and were not homogeneously verified with a standardized procedure. Nevertheless, the frequency of response to therapies was similar to what reported in the literature ${ }^{21-26}$, supporting the fact that this study was performed in centers with expertise on this disease. Despite these limitations, the observational nature of this study, in addition to the large number of our collected sample, is a unique opportunity to provide information on the clinical features of patients with CIDP and to verify the usefulness and the critical points of currently used diagnostic criteria in a real-world clinical practice setting. 


\section{Italian CIDP Database study group}

Pietro Emiliano Doneddu, Giuseppe Liberatore, Francesca Gallia, and Eduardo Nobile-Orazio from the Department of Medical Biotechnology and Translational Medicine, Neuromuscular and Neuroimmunology Service, Humanitas Clinical and Research Institute, Milan University, Rozzano, Milan, Italy; Erdita Peci and Dario Cocito from the Department of Neuroscience, University of Turin, Turin, Italy. Stefano Tronci and Raffaella Fazio from the Division of Neuroscience, Department of Neurology, Institute of Experimental Neurology (INSPE), San Raffaele Scientific Institute, Milan, Italy; Fiore Manganelli, Lucio Santoro and Emanuele Spina from the Department of Neuroscience, Reproductive Sciences and Odontostomatology, University of Naples 'Federico II', Naples, Italy; Marta Ruiz and Chiara Briani from the Neurology Unit, Department of Neuroscience, University of Padua, Padua, Italy. Stefano Cotti Piccinelli and Massimiliano Filosto from the Center for Neuromuscular Diseases and Neuropathies, Unit of Neurology ASST 'Spedali Civili', University of Brescia, Brescia, Italy; Alessandro Beronio and Luana Benedetti from the Neurology Unit, Sant'Andrea Hospital, La Spezia, Italy; Antonio Toscano, Luca Gentile and Anna Mazzeo from the Department of Clinical and Experimental Medicine, Unit of Neurology, University of Messina, Messina, Italy; Giorgia Mataluni and Girolama Alessandra Marfia from the Disimmune Neuropathies Unit, Department of Systems Medicine, Tor Vergata University of Rome, Rome, Italy; Laura Piccolo, Ilaria Callegari and Andrea Cortese from the IRCCS Foundation C. Mondino National Neurological Institute, Pavia, Italy; Giuseppe Cosentino and Brigida Fierro from the Department of Experimental BioMedicine and Clinical Neurosciences (BioNeC), University of 
Palermo, Palermo, Italy; Verrengia Elena Pinuccia and Stefano Jann from the Department of Neuroscience, Niguarda Ca' Granda Hospital, Milan, Italy; Elisa Bianchi and Ettore Beghi from the Laboratorio di Malattie Neurologiche, IRCCS-Istituto Mario Negri, Milan, Italy; Angelo Maurizio Clerici from the Neurology Unit, Circolo \& Macchi Foundation Hospital, Insubria University, DBSV, Varese, Italy; Federica Scrascia and Marinella Carpo from the ASST Bergamo OvestOspedale Treviglio, Treviglio, Italy; Martina Garnero and Angelo Schenone from the Department of Neuroscience, Rehabilitation, Ophthalmology, Genetics, Maternal and Child Health, University of Genoa and IRCCS AOU San Martino-IST, Genoa, Italy; Marco Luigetti and Mario Sabatelli from the Department of Neurology, Catholic University of Sacred Heart, Rome, Italy; Patrizia Dacci and Giuseppe Lauria from the Unit of Neuroalgology, IRCCS Foundation "Carlo Besta" Neurological Institute, Milan, Italy; Luca Leonardi and Giovanni Antonini from the Unit of Neuromuscular Diseases, Department of Neurology Mental Health and Sensory Organs (NESMOS), Faculty of Medicine and Psychology, 'Sapienza' University of Rome, Sant'Andrea Hospital, Rome, Italy; Tiziana Rosso from the Azienda UL.SS. 8 Asolo, Castelfranco Veneto, Italy;

Erika Schirinzi and Gabriele Siciliano from the Neurology Unit, Department of Clinical and Experimental Medicine, University of Pisa, Pisa, Italy; Claudia Balducci and Guido Cavaletti from the School of Medicine and Surgery and Experimental Neurology Unit, University of MilanoBicocca, Monza, Italy.

\section{Contributors}

Giuseppe Liberatore contributed to the conception of the research project, reviewed and commented on the statistical analysis, wrote the first draft of the report, and reviewed the report. Eduardo Nobile Orazio conceived, organized and designed the study, reviewed and commented on the 
statistical analysis, wrote the first draft of the report, reviewed the report. All the other authors contributed to the organization, and execution of the research project, reviewed and commented the report.

\section{Funding}

The study was supported by a Grant from Regione Lombardia, Italy, for patients from this Region and subsequently extended to other Italian Centers. The study was also supported by unrestricted grants from Kedrion Biopharma (Italy), CSL Behring (Italy), Humanitas Clinical and Research Institute (Milan, Italy), and GBS-CIDP Foundation International (USA). The funders had no role in study design, data collection and analysis, decision to publish, or preparation of the manuscript.

\section{Conflicting Interests}

Dr. Eduardo Nobile-Orazio reported personal fees for Advisory or Scientific Board from Kedrion, Italy, Baxter, Italy, Novartis, Switzerland, CSL-Behring, Italy, LFB, France, Astellas, the Netherlands, outside the submitted work and travel grants to attend Scientific Meeting from Baxter, Grifols, Kedrion, and Novartis, Italy. Dr. Pietro Emiliano Doneddu reported travel grants to attend scientific meetings from CSL Behring and Kedrion. Dr. Giuseppe Liberatore reported travel grants to attend scientific meetings from CSL Behring and Kedrion. Dr. Dario Cocito reported honoraria for lecturing from Shire, CSL Behring, and Kedrion and travel grants to attend scientific meeting from Shire, Kedrion, and CSL Behring. Dr. Erdita Peci reported travel grants to attend scientific meetings from CSL Behring. Dr. Raffaella Fazio has served on scientific advisory boards for CSL Behring and has received travel grants from Kedrion and CSL Behring to attend scientific meeting. Dr. Marinella Carpo reported travel grants to attend scientific meetings from Kedrion. Dr. Anna Mazzeo reported travel grants from Kedrion and CSL Behring to attend scientific meeting. Dr. 
Chiara Briani has served on scientific advisory boards for Pfizer and has received travel grants from Kedrion and CSL Behring to attend scientific meeting. Dr. Giuseppe Cosentino reported travel grants to attend scientific meetings from CSL Behring and Kedrion. Dr. Andrea Cortese reported travel grants to attend scientific meetings from Kedrion. Dr. Lucio Santoro reported personal fees for scientific events from CSL Behring and has received travel grants to attend scientific meetings from CSL Behring and Kedrion. Dr. Fiore Manganelli reported personal fees for scientific events from CSL Behring and has received travel grants to attend scientific meetings from CSL Behring and Kedrion. Dr. Guido Cavaletti reported honoraria for lecturing and travel grants to attend scientific meetings from Kedrion. Dr. Massimiliano Filosto has served on scientific advisory boards for CSL Behring and Sarepta Therapeutics and has received travel grants from Sanofi Genzyme, Kedrion, Baxter and CSL Behring to attend scientific meeting. Dr. Stefano Jann reported research grants from Grifols, outside this work, and travel grants from Grifols and Kedrion. Dr. Girolama Alessandra Marfia reported consulence fees and travel fundings from CSL Behring, Kedrion, Shire and Grifols. Dr. Giovanni Antonini reported honoraria for lecturing from Kedrion and Sanofigenzyme, travel grants from Kedrion, Sanofi-Genzyme and LJ Pharma. Dr. Giorgia Mataluni reported consulence fees and travel fundings from CSL Behring, Kedrion, Shire and Grifols. All the other authors have no conflict of interest.

This article is protected by copyright. All rights reserved. 


\section{REFERENCES}

1. Lunn MP, Manji H, Choudhary PP, Hughes RA, Thomas PK. Chronic inflammatory demyelinating polyradiculoneuropathy: a prevalence study in south east England. J Neurol Neurosurg Psychiatry 1999;66:677-680.

2. Laughlin RS, Dyck PJ, Melton LJ III, Leibson C, Ransom J, Dyck PJ. Incidence and prevalence of CIDP and the associations with diabetes mellitus. Neurology 2009;73:39-45.

3. Koller H, Kieseier BC, Jander S, Hartung HP. Chronic inflammatory demyelinating polyneuropathy. N Engl J Med 2005;352:1343-1356.

4. Nobile Orazio E. Chronic inflammatory demyelinating polyradiculoneuropathy and variants: where we are and where we should go. J Peripher Nerv Syst. 2014;19:2-13.

5. Lehmann HC, Broke N, Kuwabara S. Chronic inflammatory demyelinating polyneuropathy: update on diagnosis, immunopathogenesis and treatment. J Neurol Neurosurg Psychiatry. 2019 Apr 16. pii: jnnp-2019-320314. doi: 10.1136/jnnp-2019-320314. [Epub ahead of print] .

6. Ad Hoc Subcommittee of the American Academy of Neurology AIDS Task Force. Criteria for diagnosis of chronic inflammatory demyelinating polyneuropathy (CIDP). Neurology 1991;41:617-618.

7. Koski CL, Baumgarten M, Magder LS, Barohn RJ, Goldstein J, Graves M, et al. Derivation and validation of diagnostic criteria for chronic inflammatory demyelinating polyneuropathy. J Neurol Sci 2009;277:1-8.

8. Hughes RA, Bouche P, Cornblath DR, Evers E, Hadden RD, Hahn AF, et al. European Federation of Neurological Societies/Peripheral Nerve Society guideline on management of chronic inflammatory demyelinating polyneuropathy: report of a joint task force of the 
European Federation of Neurological Societies and the Peripheral Nerve Society. Eur J Neurol 2006;13:326-332.

9. Van den Bergh PY, Hadden RD, Bouche P, Cornblath DR, Hanh AF, Illa I, et al. European Federation of Neurological Societies/Peripheral Nerve Society guideline on management of chronic inflammatory demyelinating polyneuropathy: report of a joint task force of the European Federation of Neurological Societies and the Peripheral Nerve Society-First Revision. Eur J Neurol 2010;17:356-363.

10. Breiner A, Brannagan TH III. Comparison of sensitivity and specificity among 15 criteria for chronic inflammatory demyelinating polyneuropathy. Muscle Nerve 2014 Jul;50:40-46.

11. Rajabally YA, Nicolas G, Piéret F, Bouche P, Van den Bergh PY. Validity of diagnostic criteria for chronic inflammatory demyelinating polyneuropathy: a multicentre European study. J Neurol Neurosurg Psychiatry 2009;80:1364-1368.

12. Kleyweg RP, van der Meché FG, Schmitz PI. Interobserver agreement in the assessment of muscle strength and functional abilities in Guillain-Barré syndrome. Muscle Nerve 1991; 14: 1103-09.

13. Merkies ISJ, Schmitz PIM, van der Meché FGA, van Doorn PA for the European neuropathy Cause and treatment Group. Psychometric evaluation of a new sensory scale in immunemediated polyneuropathies. Neurology 2000; 54: 943-9.

14. Hughes RAC, Bensa S, Willison HJ, Van den Bergh P, Comi G, Illa I, et al. Randomized controlled trial of intravenous immunoglobulin versus oral prednisolone in chronic inflammatory demyelinating polyradiculoneuropathy. Ann Neurol 2001; 50: 195-201.

This article is protected by copyright. All rights reserved. 
15. Breiner A, Moher D, Brooks J, Cheng W, Hegen H, Deisenhammer F, et al. Adult CSF total protein upper reference limits should be agepartitioned and significantly higher than $0.45 \mathrm{~g} / \mathrm{L}$ : a systematic review. J Neurol 2019;266(3):616-624.

16. Sinnreich M, Klein CJ, Daube JR, Engelstad J, Spinner RJ, Dyck PJ. Chronic immune sensory polyradiculopathy. A possibly treatable sensory ataxia. Neurology 2004; 63: 1662-1669.

17. Doneddu PE, Cocito D, Manganelli F, Fazio R, Briani C, Filosto M, et al. Atypical CIDP: diagnostic criteria, progression, and treatment response. Data from the Italian CIDP Database. J Neurol Neurosurg Psychiatry 2019; 90: 125-132.

18. Hattori N, Misu K, Koike H, Ichimura M, Nagamatsu M, Hirayama M, et al. Age of onset influences clinical features of chronic inflammatory demyelinating polyneuropathy. J Neurol Sci 2001;184(1): 57-63.

19. Ruts L, Drenthen J, Jacobs BC, van Doorn PA. Distinguishing acute-onset CIDP from fluctuating Guillain-Barré syndrome. Neurology 2010; 74: 1680-86.

20. Fokke C, van den Berg B, Drenthen J, Walgaard C, van Doorn PA, Jacobs BC, et al. Diagnosis of Guillain-Barré syndrome and validation of Brighton criteria. Brain 2014; 137: 33-43.

21. Cocito D, Paolasso I, Antonini G, Benedetti L, Briani C, Comi C, et al. A nationwide retrospective analysis on the effect of immune therapies in patients with chronic inflammatory demyelinating polyradiculoneuropathy. Eur J Neurol 2010;17:289-294.

22. Eftimov F, Winer JB, Vermeulen M, de Haan R, van Schaik IN. Intravenous immunoglobulin for chronic inflammatory demyelinating polyradiculoneuropathy. Cochrane Database Syst Rev 2013;12:CD001797.

23. Mehndiratta MM, Hughes RA. Corticosteroids for chronic inflammatory demyelinating polyradiculoneuropathy. Cochrane Database Syst Rev 2012;8:CD002062. 
24. Mahdi-Rogers M, Swan AV, van Doorn PA, Hughes RA. Immunomodulatory treatment other than corticosteroids, immunoglobulin and plasma exchange for chronic inflammatory demyelinating polyradiculoneuropathy. Cochrane Database Syst Rev 2013;6:CD003280.

25. van Schaik IN, Bril V, van Geloven N, Hartung HP, Lewis RA, Sobue G, et al. Subcutaneous immunoglobulin for maintenance treatment in chronic inflammatory demyelinating polyneuropathy (PATH): a randomised, double-blind, placebo-controlled, phase 3 trial. Lancet Neurol. 2018; 17(1): 35-46.

26. Nobile-Orazio E, Cocito D, Jann S, Uncini A, Beghi E, Messina P, et al. Intravenous immunoglobulin versus intravenous methylprednisolone for chronic inflammatory demyelinating polyradiculoneuropathy: a randomised controlled trial. Lancet Neurol 2012, 11: 493-502

Table 1: Demographic, clinical and laboratory/instrumental test results in patients fulfilling EFNS/PNS CIDP criteria.

\begin{tabular}{|c|c|}
\hline & $\begin{array}{c}\text { EFNS/PNS CIDP } \\
(n=437)\end{array}$ \\
\hline Gender (\%F) & $35.4 \%$ \\
\hline Age at onset (ys, mean \pm SD) & $49.9 \pm 16.91$ \\
\hline Age at enrollment (ys, mean \pm SD) & $58.1 \pm 15.29$ \\
\hline Disease Duration (ys, mean \pm SD) & $8.1 \pm 8.33$ \\
\hline INCAT at enrollment ( \pm SD) & $2.59 \pm 2.01$ \\
\hline \multicolumn{2}{|l|}{ Symptoms at onset: } \\
\hline Motor & $67(15.4 \%)$ \\
\hline Sensory & $137(31.4 \%)$ \\
\hline Sensorimotor & $225(51.4 \%)$ \\
\hline Pain & $4(0.9 \%)$ \\
\hline Diplopia & $3(0.7 \%)$ \\
\hline Ataxia & $1(0.2 \%)$ \\
\hline \multicolumn{2}{|l|}{ Symptoms at enrollment: } \\
\hline Motor & $393(89.9 \%)$ \\
\hline Sensory & $419(95.6 \%)$ \\
\hline Fatigue & $235(53.8 \%)$ \\
\hline Pain & $138(31.6 \%)$ \\
\hline
\end{tabular}




\begin{tabular}{|c|c|}
\hline \\
\hline Cramps & 65 (14.9\%) \\
\hline Ataxia & $127(29.1 \%)$ \\
\hline Tremor & $51(11.7 \%)$ \\
\hline Total cranial nerves & $89(20.4 \%)$ \\
\hline Diplopia & $35(8.0 \%)$ \\
\hline Facial palsy & $27(6.2 \%)$ \\
\hline Dysphagia/dysphonia & $35(8 \%)$ \\
\hline Other Cranial nerves & $16(3.7 \%)$ \\
\hline Dysautonomia & 32 (7.3\%) \\
\hline \multicolumn{2}{|l|}{ Clinical phenotype: } \\
\hline Typical/Atypical (\%Atypical) & $353 / 84(19.2 \%)$ \\
\hline DADS & $32(7.3 \%)$ \\
\hline Sensory & $18(4.1 \%)$ \\
\hline Motor & $14(3.2 \%)$ \\
\hline Lewis-Sumner & $16(3.7 \%)$ \\
\hline Focal & $4(0.9 \%)$ \\
\hline \multicolumn{2}{|l|}{ Disease Course: } \\
\hline Progressive/Relapsing (\% Relapsing) & $204 / 228(52.8 \%)$ \\
\hline Acute Onset & $41(9.5 \%)$ \\
\hline \multicolumn{2}{|l|}{ Supportive criteria: } \\
\hline - Increased CSF proteins/tested & 230/336 (68.5\%) \\
\hline mean $(\mathrm{mg} / \mathrm{dl})$ & 106.1 \\
\hline CSF proteins $\geq 80 \mathrm{mg} / \mathrm{dl}$ & $158(47.0 \%)$ \\
\hline $\begin{array}{ll}\text { - } & \text { Sensory nerve } \\
\text { demyelination/tested }\end{array}$ & 150/394 (38.1\%) \\
\hline $\begin{array}{l}\text { - Demyelination-Inflammation at } \\
\text { nerve biopsy/tested }\end{array}$ & $21 / 35(60 \%)$ \\
\hline $\begin{array}{r}\text { - US-MRI abnormalities/tested } \\
\text { US abn./tested } \\
\text { MRI abn./tested }\end{array}$ & $\begin{array}{l}50 / 65(76.9 \%) \\
37 / 47(78.7 \%) \\
23 / 34(67.6 \%)\end{array}$ \\
\hline Response to overall therapies/treated & 338/394 (85.8\%) \\
\hline Response to IVIg/treated & 244/334 (73.1\%) \\
\hline Response to steroids/treated & $122 / 235$ (51.9\%) \\
\hline Response to Plasma exchange/treated & 25/43 (58.1\%) \\
\hline Response to SCIg/treated & 28/65 (43\%) \\
\hline Response to Immunosuppressants/treated & $31 / 97$ (31.9\%) \\
\hline
\end{tabular}

This article is protected by copyright. All rights reserved. 
Table 2: Comparison of demographic, clinical and laboratory/instrumental test results in patients with progressive or relapsing CIDP.

\begin{tabular}{|c|c|c|c|}
\hline & $\begin{array}{l}\text { RELAPSING } \\
(\mathrm{n}=228)\end{array}$ & $\begin{array}{c}\text { PROGRESSIVE } \\
(n=204)\end{array}$ & P-value \\
\hline Gender (\%F) & $35.1 \%$ & $35.7 \%$ & $>0.1$ \\
\hline Age at onset (ys, mean \pm SD) & $47.4 \pm 16.77$ & $52.8 \pm 16.74$ & 0.001 \\
\hline Age at enrollment (ys, mean \pm SD) & $56.1 \pm 15.17$ & $60.6 \pm 15.03$ & 0.003 \\
\hline Disease Duration (ys, mean \pm SD) & $8.5 \pm 9.12$ & $7.6 \pm 7.43$ & $>0.1$ \\
\hline INCAT at enrollment $( \pm S D)$ & $2.3 \pm 2.05$ & $2.9 \pm 1.91$ & $<0.001$ \\
\hline
\end{tabular}

This article is protected by copyright. All rights reserved. 
Symptoms at onset:

\begin{tabular}{|c|c|c|c|}
\hline Fatigue & 0 & $1(0.5 \%)$ & NA \\
\hline \multicolumn{4}{|l|}{ Symptoms at enrollment: } \\
\hline Motor & $203(89.0 \%)$ & $187(91.7 \%)$ & $>0.1$ \\
\hline Sensory & 219 (96.1\%) & 197 (96.6\%) & $>0.1$ \\
\hline Fatigue & $124(54.4 \%)$ & $109(53.4 \%)$ & $>0.1$ \\
\hline Pain & $71(31.1 \%)$ & $65(31.9 \%)$ & $>0.1$ \\
\hline Cramps & $32(14.0 \%)$ & $33(16.2 \%)$ & $>0.1$ \\
\hline Ataxia & $72(31.6 \%)$ & $55(27.0 \%)$ & $>0.1$ \\
\hline Tremor & $22(9.6 \%)$ & $28(13.7 \%)$ & $>0.1$ \\
\hline Cranial nerves impairment & $48(21.1 \%)$ & $41(20.1 \%)$ & $>0.1$ \\
\hline Dysautonomia & $18(7.9 \%)$ & $14(6.9 \%)$ & $>0.1$ \\
\hline \multicolumn{4}{|l|}{ Clinical phenotype: } \\
\hline Typical/Atypical (\%Atypical) & $186 / 42(18.4 \%)$ & $164 / 40(19.6 \%)$ & $>0.1$ \\
\hline Acute onset & $28(12.3 \%)$ & $13(6.4 \%)$ & 0.048 \\
\hline \multicolumn{4}{|l|}{ Supportive criteria: } \\
\hline - $\quad$ Increased CSF proteins/tested & $120 / 177(67.8 \%)$ & $104 / 153(67.9 \%)$ & $>0.1$ \\
\hline mean (mg/dl) & 105.9 & 98 & $>0.1$ \\
\hline CSF proteins $\geq 80 \mathrm{mg} / \mathrm{dl}$ & $82(46.3 \%)$ & 72 (47.1\%) & $>0.1$ \\
\hline $\begin{array}{ll}\text { - Sensory nerve } \\
\text { demyelination/tested }\end{array}$ & 70/192 (36.5\%) & 66/182 (36.3\%) & $>0.1$ \\
\hline Response to overall therapies/treated & $178 / 205(86.8 \%)$ & 158/186 (84.9\%) & $>0.1$ \\
\hline Response to IVIg/treated & 121/177 (68.4\%) & 122/155 (78.7\%) & 0.035 \\
\hline Response to steroids/treated & 73/140 (52.1\%) & 49/94 (52.1\%) & $>0.1$ \\
\hline
\end{tabular}

Table 3: Comparison of demographic, clinical and laboratory/instrumental test results in patients with acute-onset or not acute-onset CIDP.

$\begin{array}{clc}\underset{\substack{\text { ACUTE-ONSET CIDP } \\(\mathrm{n}=41)}}{ } & \begin{array}{l}\text { NOT ACUTE- } \\ \text { ONSET CIDP }\end{array} & \text { P-value }\end{array}$

This article is protected by copyright. All rights reserved. 


\begin{tabular}{|c|c|c|c|}
\hline & & $(n=391)$ & \\
\hline Gender (\%F) & $34.1 \%$ & $35.5 \%$ & $>0.1$ \\
\hline Age at onset (ys, mean \pm SD) & $45.2 \pm 19.27$ & $50.3 \% \pm 17.87$ & 0.063 \\
\hline Age at enrollment (ys, mean \pm SD) & $52.7 \pm 17.46$ & $58.6 \pm 16.23$ & 0.017 \\
\hline Disease Duration (ys, mean \pm SD) & $7.6 \pm 9.97$ & $8.1 \pm 8.43$ & $>0.1$ \\
\hline INCAT at enrollment $( \pm S D)$ & $3.2 \pm 2.47$ & $2.5 \pm 2.78$ & 0.048 \\
\hline \multicolumn{4}{|l|}{ Symptoms at onset: } \\
\hline Motor & $7(17.1 \%)$ & $62(15.8 \%)$ & $>0.1$ \\
\hline Sensory & 7 (17.1\%) & $138(35.2 \%)$ & 0.035 \\
\hline Sensorimotor & $25(60.9 \%)$ & $184(47 \%)$ & $>0.1$ \\
\hline Pain & 0 & $4(0.1 \%)$ & NA \\
\hline Diplopia & $2(4.9 \%)$ & $1(0.2 \%)$ & NA \\
\hline Ataxia & 0 & $1(0.2 \%)$ & NA \\
\hline Fatigue & 0 & $1(0.2 \%)$ & NA \\
\hline \multicolumn{4}{|l|}{ Symptoms at enrollment: } \\
\hline Motor & $41(100 \%)$ & $348(91 \%)$ & 0.014 \\
\hline Sensory & 39 (95.1\%) & 375 (95.9\%) & $>0.1$ \\
\hline Fatigue & $24(58.5 \%)$ & $210(53.7 \%)$ & $>0.1$ \\
\hline Pain & 17 (41.5\%) & $121(30.9 \%)$ & $>0.1$ \\
\hline Cramps & $2(4.9 \%)$ & $63(16.3 \%)$ & 0.069 \\
\hline Ataxia & $12(29.3 \%)$ & $114(29.1 \%)$ & $>0.1$ \\
\hline Tremor & $9(22 \%)$ & $43(10.9 \%)$ & 0.076 \\
\hline Cranial nerves impairment & $21(51.2 \%)$ & $68(17.4 \%)$ & $<0.001$ \\
\hline Dysautonomia & $5(12.2 \%)$ & $26(6.6 \%)$ & $>0.1$ \\
\hline \multicolumn{4}{|l|}{ Clinical phenotype: } \\
\hline Typical/Atypical (\%Atypical) & $38 / 3(7.3 \%)$ & 315/76 (24.1\%) & 0.057 \\
\hline \multicolumn{4}{|l|}{ Supportive criteria: } \\
\hline - Increased CSF proteins/tested & 28/33 (90.9\%) & 202/313 (64.5\%) & 0.019 \\
\hline mean (mg/dl) & 125.1 & 120.2 & $>0.1$ \\
\hline CSF proteins $\geq 80 \mathrm{mg} / \mathrm{dl}$ & $21(63.4 \%)$ & $120(38.3 \%)$ & 0.008 \\
\hline $\begin{array}{ll}\text { - } & \text { Sensory nerve } \\
\text { demyelination/tested }\end{array}$ & 14/32 (43.7\%) & 128/356 (35.9\%) & $>0.1$ \\
\hline Response to overall therapies/treated & 38/39 (97.4\%) & 292/355 (82.2\%) & 0.025 \\
\hline Response to IVIg/treated & 30/35 (85.7\%) & 213/299 (71.2\%) & 0.076 \\
\hline Response to steroids/treated & $14 / 21(67.7 \%)$ & 108/214 (50.4\%) & $>0.1$ \\
\hline
\end{tabular}

This article is protected by copyright. All rights reserved. 
Figure 1 Flow-chart of the cohort.

CMT: Charcot-Marie-Tooth disease

MAG: Myelin-associated glycoprotein

NCS: Nerve conduction studies

Figure 2 Distribution of abnormal results in 85 patients with NCS-possible or NCS-probable CIDP who improved (78 patients) or not (seven patients) the certainty of their diagnosis with supportive criteria.

CSF: Cerebro-Spinal Fluid

SAP: Sensory Action Potential

SSEP: Somato-Sensory Evoked Potentials

MRI: Magnetic Resonance Imaging

US: Ultrasound exam

This article is protected by copyright. All rights reserved. 


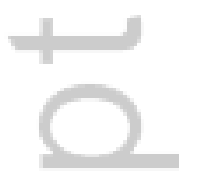

4 


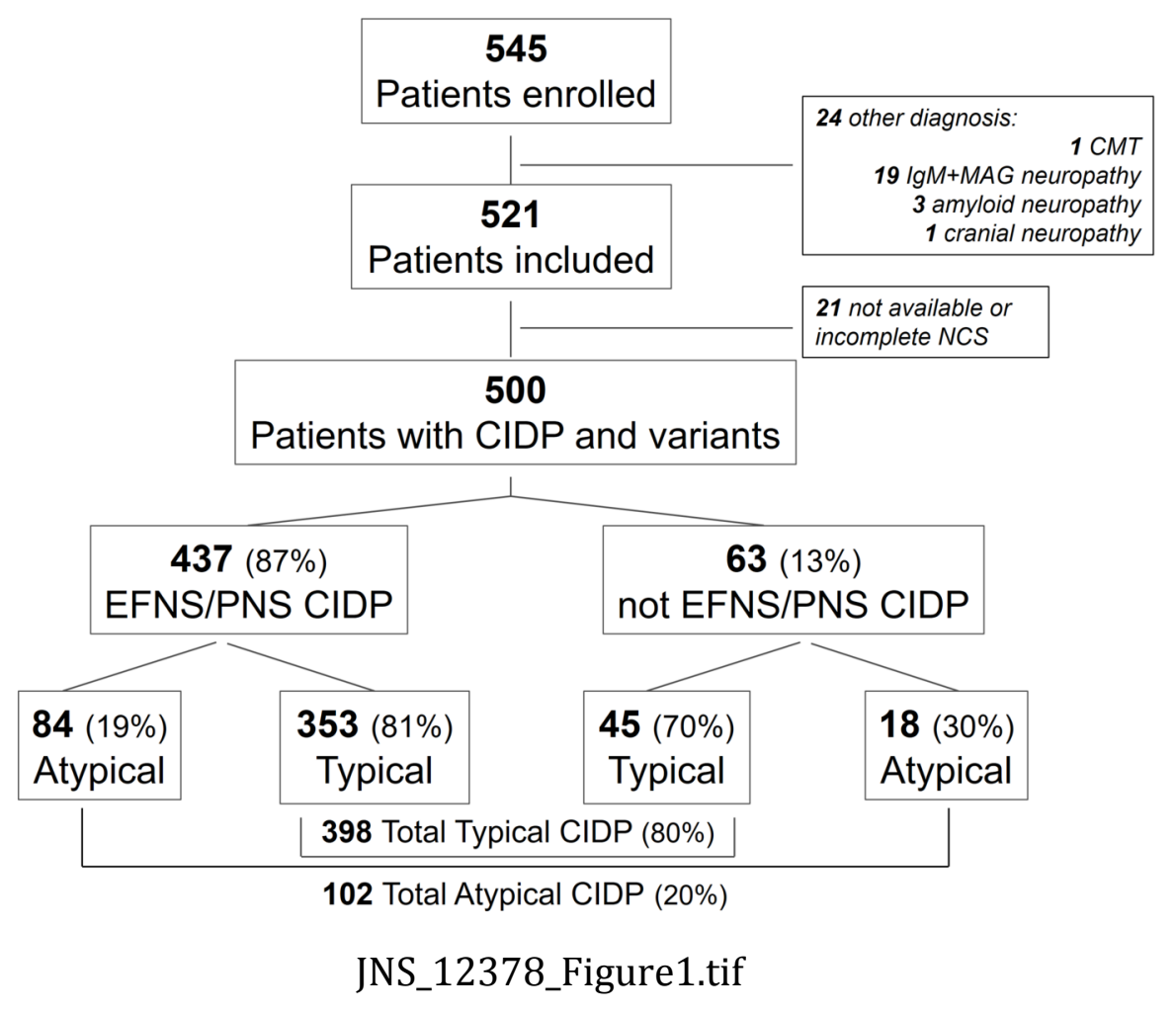

This article is protected by copyright. All rights reserved. 


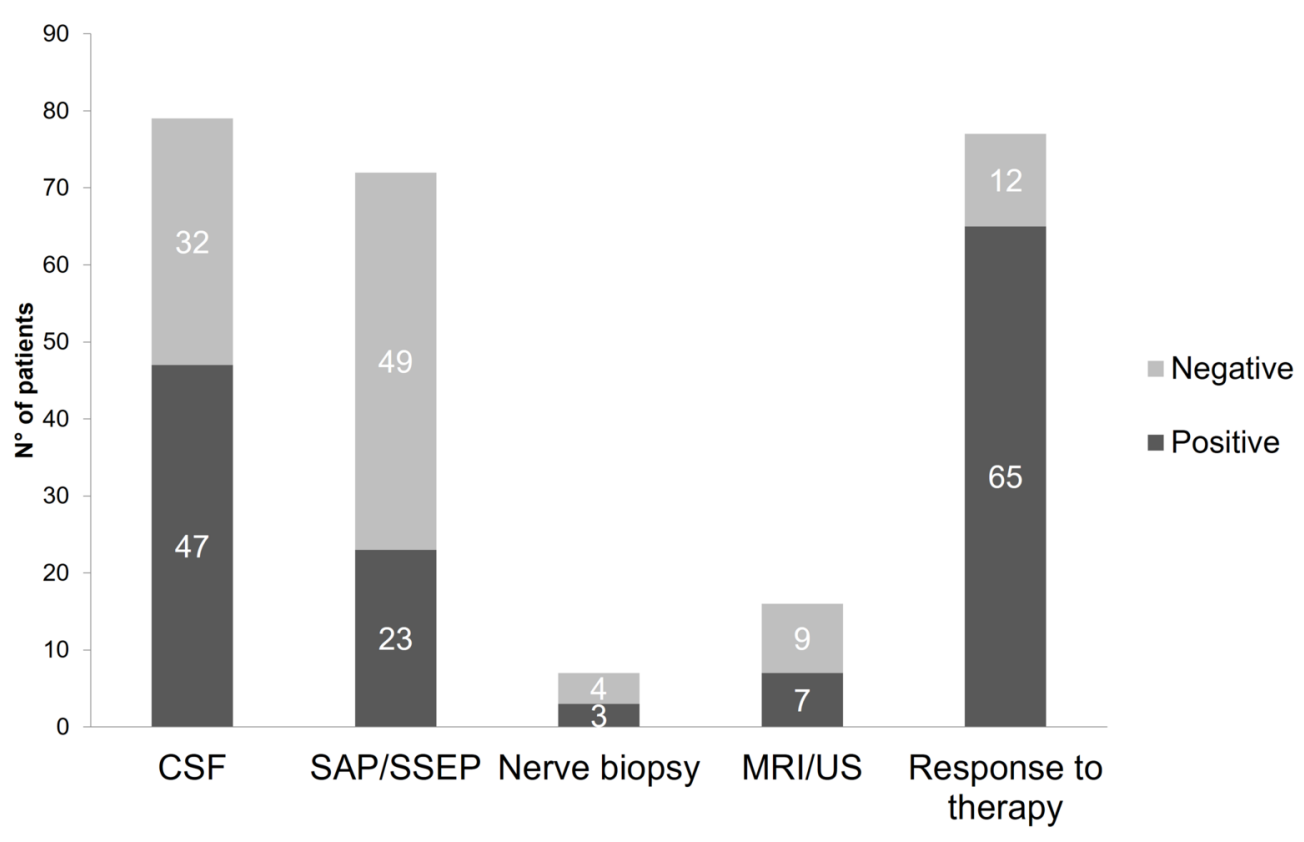

JNS_12378_Figure2rev.tif

This article is protected by copyright. All rights reserved. 